\title{
Maximal Chains of Prime Ideals of Different Lengths in Unique Factorization Domains
}

\author{
S. Loepp and Alex Semendinger
}

January 10, 2019

\begin{abstract}
We show that, given integers $n_{1}, n_{2}, \ldots, n_{k}$ with $2<n_{1}<n_{2}<\cdots<n_{k}$, there exists a local (Noetherian) unique factorization domain that has maximal chains of prime ideals of lengths $n_{1}, n_{2}, \ldots, n_{k}$ which are disjoint except at their minimal and maximal elements. In addition, we demonstrate that unique factorization domains can have other unusual prime ideal structures.
\end{abstract}

\section{Introduction}

One important open question in commutative algebra is: given a partially ordered set $X$, is $X$ isomorphic to the prime spectrum of some Noetherian ring? While this question itself has not been answered, progress towards it has been made. For instance, in [6], Hochster answered the question of when $X$ is isomorphic to the prime spectrum of a possibly nonNoetherian commutative ring.

One approach to the problem is to consider what posets may arise as finite subsets of Spec $R$ for a Noetherian ring $R$. It was once thought that these would necessarily possess "nice" properties: most notably, it was at one point thought that all Noetherian rings must be catenary. This was disproven in 1956 in [9], when Nagata constructed a noncatenary 
Noetherian integral domain. In 1979, Heitmann proved a much stronger result: in [4], he showed that, for every finite partially ordered set $X$, there exists a Noetherian domain $R$ such that $X$ can be embedded into $\operatorname{Spec} R$ with an embedding that preserves saturated chains.

It is natural to ask if Heitmann's theorem holds for other classes of rings, such as Noetherian unique factorization domains (UFDs). One might expect that the additional structure of a UFD would place restrictions on its prime ideal structure. More precisely, we ask the following open question:

Question 1.1. For every finite partially ordered set $X$, does there exist a Noetherian UFD A such that $X$ can be embedded into Spec $A$ with an embedding that preserves saturated chains?

It has long been thought that this question could be answered in the negative. For instance, after 1956, it was conjectured that all Noetherian unique factorization domains (and more generally, all integrally closed domains) must be catenary. This was disproved by Heitmann in [5], in which he constructed a noncatenary Noetherian UFD. This was the only example of a noncatenary Noetherian UFD in the literature until 2017, when Avery, et. al. characterized the completions of noncatenary UFDs in [1], which generated a new class of examples. As a corollary of this result ([1], Proposition 4.2), Avery, et. al. showed that there is no limit on "how noncatenary" a local UFD can be, in the sense that it can posess maximal chains of prime ideals with an arbitrarily large difference in lengths. Inasmuch as this result furnishes more examples of noncatenary Noetherian UFDs, it provides mild evidence in favor of an affirmative answer to Question 1.1 .

In this paper, although we do not provide an answer to Question 1.1, we further develop the techniques from [1] and give explicit examples of noncatenary partially ordered sets which can be embedded in a saturated way into the prime spectrum of some Noetherian UFDs. As a result, we not only greatly expand our collection of examples of noncatenary local UFDs, but we also provide additional evidence in favor of the analogue of Heitmann's 1979 theorem 
holding for UFDs.

Our main result (Theorem 3.4) is that, given integers $n_{1}, n_{2}, \ldots, n_{k}$, with $2<n_{1}<n_{2}<$ $\cdots<n_{k}$, there exists a local (Noetherian) unique factorization domain that has maximal chains of prime ideals of lengths $n_{1}, n_{2}, \ldots, n_{k}$ which are disjoint except at their minimal and maximal elements. To prove this result, we first start with a complete local ring $T$ that has maximal chains of prime ideals of lengths $n_{1}, n_{2}, \ldots, n_{k}$. We then use the construction in [5] to find a local UFD $A$ whose completion with respect to its maximal ideal is $T$ and such that the intersections of the chains of prime ideals of $T$ with $A$ results in maximal chains in $A$ of lengths $n_{1}, n_{2}, \ldots, n_{k}$. Finally, we show that, after some adjustment of the height one prime ideals in the chains, the chains are disjoint.

\section{Preliminaries}

Throughout this paper, all rings are assumed to be commutative with unity. Additionally, the symbol $\subset$ will indicate strict containment.

Definition. If $R$ is a Noetherian ring with exactly one maximal ideal $M$, then we say $(R, M)$ is a local ring. If $R$ is not necessarily Noetherian but has one maximal ideal, then we say it is quasi-local.

If $A$ is a local ring, then $\widehat{A}$ will denote the completion of $A$ with respect to its maximal ideal.

In order to formally state the motivating question of our research and our results, we need to develop some terminology regarding partially ordered sets (posets).

Definition. A totally ordered subset of a partially ordered set $X$ is called a chain.

Definition. Let $x_{0}<x_{1}<\cdots<x_{n}$ be a finite chain of elements in a poset $X$. We say that this chain has length $n$. 
If the above chain has the property that, for each $i \in\{0, \ldots, n-1\}$, there are no elements $y \in X$ such that $x_{i}<y<x_{i+1}$, then we say the chain is saturated.

If the above chain is saturated and, additionally, $x_{0}$ is a minimal element of $X$ and $x_{n}$ is a maximal element, then we say the chain is maximal.

Definition. Let $X$ be a poset. The height of $x \in X$ is the length of the longest saturated chain of elements $x_{0}<x_{1}<\cdots<x$ and is denoted ht $x$. The dimension of $X$ is given by $\operatorname{dim} X:=\sup \{$ ht $x: x \in X\}$.

Recall that, when we refer to the height of a prime ideal of a ring $R$, we mean its height as a member of the poset (Spec $R, \subseteq$ ). Furthermore, $\operatorname{dim} R=\operatorname{dim} \operatorname{Spec} R$ and is the Krull dimension of the ring $R$.

Definition. We say a poset $X$ is catenary if, for any $x, y \in X$ such that $x<y$, all saturated chains of elements between $x$ and $y$ have the same length.

This definition is usually applied to rings, where we say a ring $R$ is catenary precisely when the poset $(\operatorname{Spec} R, \subseteq$ ) is catenary.

Definition. Let $X$ and $Y$ be posets. An injective order-preserving function $\phi: X \rightarrow Y$ will be called a saturated embedding if $\phi$ sends saturated chains in $X$ to saturated chains in $Y$. If, additionally, $\operatorname{dim}(X)=\operatorname{dim}(\phi(X))$, we will say $\phi$ is a dimension-preserving saturated embedding.

Our motivating question can now be formally stated: for which partially ordered sets $X$ does there exist a local UFD $A$ and a dimension-preserving saturated embedding from $X$ to Spec $A$ ? In the next section, we show that the set of posets for which this can be done is larger than previously known.

We now state results which will be used in later proofs. First, we present two very useful prime avoidance lemmas from [5] and a "cardinality" lemma from [2]. 
Lemma 2.1. ([5], Lemma 2) Let $(T, M)$ be a complete local ring, $C$ a countable set of prime ideals of $T$ such that $M \notin C$, and $D$ a countable set of elements of $T$. If I is an ideal of $T$ which is contained in no single $P \in C$, then $I \nsubseteq \bigcup\{(r+P): P \in C, r \in D\}$.

Lemma 2.2. ([5], Lemma 3) Let $(T, M)$ be a local ring. Let $C \subset \operatorname{Spec} T$, let $I$ be an ideal of $T$ such that $I \not \subset P$ for all $P \in C$, and let $D \subset T$. Suppose $|C \times D|<|T / M|$. Then $I \nsubseteq \bigcup\{(r+P): P \in C, r \in D\}$.

Lemma 2.3. (2], Lemma 2.2) Let $(T, M)$ be a complete local ring of dimension at least one. Let $P$ be a nonmaximal prime ideal of $T$. Then $|T / P|=|T| \geq|\mathbb{R}|$.

The construction in [5] starts with a subring of a complete local ring $T$ which must satisfy several properties. Any ring satisfying these properties is called an N-subring, which we now define.

Definition. Let $(T, M)$ be a complete local ring. A quasi-local subring $(R, M \cap R)$ of $T$ is an $\mathrm{N}$-subring if $R$ is a UFD and

1. $|R| \leq \sup \left(\aleph_{0},|T / M|\right)$, with equality only when $T$ is countable,

2. $Q \cap R=(0)$ for all $Q \in \operatorname{Ass} T$, and

3. if $t \in T$ is regular and $P \in \operatorname{Ass}(T / t T)$, then $\operatorname{ht}(P \cap R) \leq 1$.

We will also need a way to adjoin elements to an $\mathrm{N}$-subring such that the resulting ring is an $\mathrm{N}$-subring as well. The following lemma allows us to do so.

Lemma 2.4. ([7, Lemma 11) Let $(T, M)$ be a complete local ring and let $R$ be an $N$-subring of T. Suppose $C \subset \operatorname{Spec} T$ satisfies the following conditions:

1. $M \notin C$,

2. $\{P \in \operatorname{Spec} T: P \in \operatorname{Ass} T / r T$ for some $0 \neq r \in R\} \subset C$, and 


\section{Ass $T \subset C$.}

Let $x \in T$ be such that $x \notin P$ and $x+P$ is transcendental over $R /(R \cap P)$ as an element of $T / P$ for every $P \in C$. Then $S=R[x]_{(R[x] \cap M)}$ is an $N$-subring of $T$ properly containing $R$ and $|S|=\sup \left\{\aleph_{0},|R|\right\}$.

Here and elsewhere, we tacitly make use of the natural injection $R /(R \cap P) \rightarrow T / P$ defined by $r+(R \cap P) \mapsto r+P$, where $R, T$, and $P$ are as defined in Lemma 2.4. Under this injection, we can think of elements of $T / P$ as being algebraic or transcendental over the ring $R /(R \cap P)$. Determinations of this sort will prove crucial in Lemma 3.3 and in Theorem 3.4 .

The construction of specific posets within Spec $A$ starts by carefully choosing chains of

prime ideals of $T=\widehat{A}$, which the next lemma allows us to do. These chains, when taken together and intersected with $A$, will essentially form the desired posets.

Lemma 2.5. ([1], Lemma 2.8) Let $(T, M)$ be a local ring with $M \notin$ Ass $T$ and let $P \in \operatorname{Min} T$ with $\operatorname{dim}(T / P)=n$. Then there exists a saturated chain of prime ideals of $T, P \subset Q_{1} \subset$ $\cdots \subset Q_{n-1} \subset M$, such that, for each $i=1, \ldots, n-1$, we have $Q_{i} \notin$ Ass $T$ and $P$ is the only minimal prime ideal of $T$ contained in $Q_{i}$.

The main result from [1] demonstrates conditions for exactly when a complete local ring is the completion of a noncatenary local UFD. Because all of the posets we will consider in this paper will be noncatenary, it will be necessary to ensure that the complete local $\operatorname{ring} T$ we begin with satisfies these conditions.

Theorem 2.6. ([1], Theorem 3.7) Let $(T, M)$ be a complete local ring. Then $T$ is the completion of a noncatenary local UFD if and only if the following conditions hold:

1. No integer of $T$ is a zero divisor,

2. $\operatorname{depth} T>1$, and 
3. There exists $P \in \operatorname{Min} T$ such that $2<\operatorname{dim}(T / P)<\operatorname{dim} T$.

We finally present a theorem from algebraic geometry which will be used to find a complete local ring with a specific minimal prime ideal structure.

Theorem 2.7. ([3], Theorem 8.18 (Bertini's Theorem)) Let $X$ be a nonsingular closed subvariety of $\mathbb{P}_{K}^{n}$, where $K$ is an algebraically closed field. Then there exists a hyperplane $H \subseteq \mathbb{P}_{K}^{n}$, not containing $X$, and such that the scheme $H \cap X$ is regular. If $\operatorname{dim} X \geq 2$, then $H \cap X$ is connected, and therefore irreducible, as well. Furthermore, the set of hyperplanes with this property forms an open dense subset of the complete linear system $|H|$, considered as a projective space.

\section{Main Theorem}

We begin by constructing a complete local ring satisfying the conditions of Theorem 2.6, as well as other desired conditions. Note that condition 6 of Lemma 3.1 implies that no integer of $T$ is a zero divisor. We then use a modified version of the construction from the proof of that theorem to obtain a local UFD with arbitrarily many disjoint chains of different lengths.

Lemma 3.1. Given any $k$-tuple $\left(n_{1}, n_{2}, \ldots, n_{k}\right)$ of distinct positive integers all greater than 2, there exists a complete local ring $T$ with minimal prime ideals $P_{1}, \ldots, P_{k}$ such that

1. $\operatorname{dim}\left(T / P_{i}\right)=n_{i}$ for each $i=1, \ldots, k$,

2. $T / P_{i}$ is a regular local ring for each $i=1, \ldots, k$,

3. $\operatorname{Min} T=\operatorname{Ass} T=\left\{P_{1}, \ldots, P_{k}\right\}$,

4. If $x \in T$ is not a zero divisor and $Q \in \operatorname{Ass}(T / x T)$ (considered as a subset of $\operatorname{Spec} T$ ), then ht $Q=1$, 
5. $\operatorname{depth} T \geq 2$, and

\section{6. $T$ contains $\mathbb{Q}$.}

Proof. Without loss of generality, suppose $n_{1}<n_{2}<\cdots<n_{k}$. Let $R=\mathbb{C}\left[x_{0}, \ldots, x_{n}\right]$, where $n=n_{k}$. By Bertini's theorem, there exists an open collection of hypersurfaces in $\mathbb{P}^{n}$ such that, if $H$ is an element of the open collection, then $H$ is a smooth, irreducible subvariety. To obtain a subvariety of dimension $n-j$ we apply the theorem $j+1$ times to obtain smooth irreducible subvarieties $H_{0} \supset\left(H_{0} \cap H_{1}\right) \supset \cdots \supset\left(H_{0} \cap \cdots \cap H_{j}\right)=H$. We then let $Q$ be the ideal corresponding to $H$; that is, $Q=I(H) \in \operatorname{Spec} R$. By Bertini's Theorem, $H$ is irreducible, hence $Q$ is a prime ideal. Furthermore, $\operatorname{dim} R / Q=n-j$. Because all of these varieties are smooth, for each resultant prime ideal $Q$, the ring $R / Q$ is regular.

We apply this result to obtain prime ideals $Q_{1}, \ldots, Q_{k}$ such that $\operatorname{dim} R / Q_{i}=n_{i}$ and $R / Q_{i}$ is regular for each $i$. To ensure that they are all incomparable, note that $Q_{i} \subseteq Q_{j}$ if and only if $V\left(Q_{j}\right) \subseteq V\left(Q_{i}\right)$. Therefore, we need only choose varieties that are not contained in one another, which is possible since the set of candidates is an open dense set, and containment is a closed condition.

Now let $I=\bigcap_{i=1}^{k} Q_{i}$ and let $S=\widehat{R} \cong \mathbb{C} \llbracket x_{0}, \ldots, x_{n} \rrbracket$, where $\widehat{R}$ denotes the completion of $R$ with respect to the maximal ideal $\left(x_{0}, \ldots, x_{n}\right)$. The desired ring is given by $T=S / I S$, where $P_{i}=Q_{i} S / I S$ (we will see shortly that this is in fact a prime ideal of $T$ ). It is clear that $T$ contains $\mathbb{Q}$.

To see that $T$ satisfies the desired properties, observe first that $Q_{1} \cap Q_{2} \cap \cdots \cap Q_{k}$ is a minimal primary decomposition of the ideal $I$ of $R$. Now, $T / P_{i}$ is a regular local ring as a result of the fact that the completion of a regular local ring is itself a regular local ring, and

$$
\left.\widehat{\left(R / Q_{i}\right.}\right) \cong S / Q_{i} S \cong \frac{S / I S}{Q_{i} S / I S} \cong T / P_{i}
$$


This also establishes that $P_{i}$ is a prime ideal (since a regular local ring is a domain) and that $\operatorname{dim}\left(T / P_{i}\right)=\operatorname{dim}\left(R / Q_{i}\right)=n_{i}$

Since $Q_{i}$ is a prime ideal of $R$ for every $i=1,2, \ldots k$, we have $\sqrt{I}=I$ and so $R / I$ is reduced. Since $R$ is excellent, $R / I$ is excellent and so its completion, $S / I S$, is also reduced. It follows that $T=S / I S$ has no embedded associated prime ideals, and so $\operatorname{Min} T=\operatorname{Ass} T$.

We now show that $\operatorname{Min} T=\left\{P_{1}, P_{2}, \ldots, P_{k}\right\}$. Suppose $P$ is a prime ideal of $S$ such that $I S \subseteq P \subseteq Q_{i} S$. Then, intersecting with $R$, we have $I \subseteq(P \cap R) \subseteq Q_{i}$. Since $Q_{i}$ is minimal over $I, P \cap R=Q_{i}$. Now, $(P \cap R) S \subseteq P \subseteq Q_{i} S$. It follows that $Q_{i} S \subseteq P \subseteq Q_{i} S$ and so $P=Q_{i} S$. Hence, $P_{i} \in \operatorname{Min} T$ for all $i=1,2, \ldots, k$. On the other hand, let $P$ be a minimal prime ideal over $I S$. Then, intersecting with $R$, we have $I \subseteq P \cap R$. Since $\operatorname{Ass}(R / I R)=\operatorname{Min}(R / I R)=\left\{Q_{1}, Q_{2}, \ldots, Q_{k}\right\}$, it must be the case that $I \subseteq Q_{i} \subseteq P \cap R$ for some $i$. It follows that $I S \subseteq Q_{i} S \subseteq(P \cap R) S \subseteq P$ and so $P=Q_{i} S$. Therefore, $\operatorname{Min} T=\left\{P_{1}, P_{2}, \ldots, P_{k}\right\}$.

Any regular ring $A$ satisfies Serre's criterion $\left(S_{2}\right)$ (by [8], Theorem 23.8), which implies that if $P \in \operatorname{Ass}(A / r A)$, for any regular $r \in A$, then ht $P \leq 1$. Suppose $x \in T$ is not a zerodivisor and let $Q \in \operatorname{Ass}(T / x T)$. Let $P_{i}$ be a minimal prime ideal of $T$ contained in $Q$. Define $\bar{T}=T / P_{i}$ and denote the image of $Q$ in $\bar{T}$ by $\bar{Q}$. Since $Q \in \operatorname{Ass}(T / x T)$, by [10], Remark 4.23, we have that $\bar{Q} \in \operatorname{Ass}(\bar{T} / x \bar{T})$. Therefore ht $Q / P_{i} \leq 1$. Since this is true for each minimal prime ideal $P_{i}$ of $T$ such that $P_{i} \subseteq Q$, we must therefore have ht $Q=1$ as desired.

To see that depth $T>1$, we will find a $T$-regular sequence of length 2 . We start by observing that, if $M$ is the maximal ideal of $T$ then $M \notin \operatorname{Ass} T$, and so there exists $x \in M$ which is not a zero divisor. If $Q \in \operatorname{Ass} T / x T$, then ht $Q=1$. However, by construction, $\operatorname{dim} T \geq 2$, so any height two prime ideal of $T$ will contain a $T / x T$-regular element, call it $y$. Then $x, y$ is a regular sequence of length 2 as desired. 
The following lemma will afford us a high degree of control over the height one elements we choose for our chains of prime ideals in the main theorem.

Lemma 3.2. Let $(A, M)$ be a local UFD with $\operatorname{dim} A>2$ and let $k$ be a positive integer. Suppose that A contains maximal chains of prime ideals

$$
(0) \subset P \subset P_{i, 2} \subset \cdots \subset P_{i, n_{i}-1} \subset M
$$

for $i=1, \ldots, k$, where $P_{a, 2} \neq P_{b, 2}$ whenever $a \neq b$. Then there exist height one prime ideals $\tilde{P}_{1}, \ldots, \tilde{P}_{k} \in \operatorname{Spec} A$ such that, for $i=1, \ldots, k, \tilde{P}_{i} \subset P_{i, 2}$ and $\tilde{P}_{a} \neq \tilde{P}_{b}$ whenever $a \neq b$.

Proof. By the Prime Avoidance Theorem, there exists $z_{i} \in P_{i, 2}$ such that $z_{i} \notin P_{j, 2}$ for $j \neq i$. Let $q_{i}$ be an irreducible factor of $z_{i}$ that is in $P_{i, 2}$. Then $q_{i} A \subset P_{i, 2}$ but $q_{i} A \not \subset P_{b, 2}$ for $b \neq i$. Now set $\tilde{P}_{i}=q_{i} A$. Then $\tilde{P}_{1}, \ldots, \tilde{P}_{k} \in \operatorname{Spec} A$ are height one prime ideals of $A$ and $\tilde{P}_{a} \neq \tilde{P}_{b}$ when $a \neq b$.

The following technical lemma will prove instrumental in the proof of the main theorem, and, along with Lemma 3.2, is among the primary techniques that allow us to have some control of the prime ideal structure of the resultant UFD.

Lemma 3.3. Let $(T, M)$ be a complete local ring as constructed in Lemma 3.1. Furthermore, let $Q \in \operatorname{Spec} T$ be such that ht $Q>1$ and let $R$ be a countable $N$-subring of $T$.

Then there exists a countable $N$-subring $S$ of $T$ such that $R \subset S$ and $S$ contains a generating set for $Q$.

Proof. Let $Q=\left(x_{1}, \ldots, x_{n}\right)$. We will find elements $\tilde{x}_{1}, \ldots, \tilde{x}_{n} \in Q$ such that $Q=\left(\tilde{x}_{1}, \ldots, \tilde{x}_{n}\right)$ and such that we have a chain of $\mathrm{N}$-subrings

$$
R=R_{0} \subset R_{1} \subset R_{2} \subset \cdots \subset R_{n}=S
$$


where $R_{i}=R_{i-1}\left[\tilde{x}_{i}\right]_{M \cap R_{i-1}\left[\tilde{x}_{i}\right]}$ for each $i=1, \ldots, n$.

We begin by finding an element $\tilde{x}_{1} \in Q$ such that $R_{1}=R_{0}\left[\tilde{x}_{1}\right]_{M \cap R_{0}\left[\tilde{x}_{1}\right]}$ is an N-subring and $Q=\left(\tilde{x}_{1}, x_{2}, \ldots, x_{n}\right)$. We can accomplish this through the use of Lemma 2.4, for which it will suffice to find an element $\tilde{x} \in Q$ such that $\tilde{x}_{1}+P$ is transcendental over $R_{0} /\left(R_{0} \cap P\right)$ as an element of $T / P$ for all $P \in C_{1}=\left\{P \in \operatorname{Spec} T: P \in \operatorname{Ass}(T / r T), 0 \neq r \in R_{0}\right\} \cup \operatorname{Ass} T$.

First, by Lemma [3.1, $M \notin C_{1}$ and $Q \not \subset P$ for all $P \in C_{1}$, so by Lemma 2.1 there exists $y_{1} \in Q$ such that $y_{1} \notin P$ for all $P \in C_{1}$. We will set $\tilde{x}_{1}=x_{1}+\alpha_{1} y_{1}$, where $\alpha_{1} \in T$ remains to be chosen.

Fix some $P \in C_{1}$. Note that $\left|R_{0} /\left(R_{0} \cap P\right)\right| \leq\left|R_{0}\right|$, and $R_{0}$ is countable. As a result, the algebraic closure of $R_{0} /\left(R_{0} \cap P\right)$ in $T / P$ is countable. However, by Lemma 2.3, $T / P$ is uncountable. Since we have chosen $y_{1} \notin P$, every distinct choice of $t+P \in T / P$ gives a different $x_{1}+t y_{1}+P$. There are thus uncountably many such choices, only countably many of which make $x_{1}+t y_{1}+P$ algebraic over $R_{0} /\left(R_{0} \cap P\right)$. In order to find a value of $\alpha_{1}$ such that $\tilde{x}_{1}+P$ is transcendental over $R_{0} /\left(R_{0} \cap P\right)$ for all $P \in C_{1}$ simultaneously, we make use of another application of Lemma 2.1. For each $P \in C_{1}$, let $D_{(P)}$ be a set consisting of one element from each coset of $T / P$ which makes $x_{1}+t y_{1}+P$ algebraic over $R_{0} /\left(P \cap R_{0}\right)$. Then set $D_{1}=\bigcup_{P \in C_{1}} D_{(P)}$. We can now use Lemma 2.1 to find $\alpha_{1} \in M$ such that $\alpha_{1} \notin \bigcup\left\{(r+P): P \in C_{1}, r \in D_{1}\right\}$. This ensures that $x_{1}+\alpha_{1} y_{1}+P$ is transcendental over $R_{0} /\left(R_{0} \cap P\right)$ for all $P \in C_{1}$, which was our goal. We can now set $\tilde{x}_{1}=x_{1}+\alpha_{1} y_{1}$ and let $R_{1}=R_{0}\left[\tilde{x}_{1}\right]_{M \cap R_{0}\left[\tilde{x}_{1}\right]}$. Note that $R_{1}$ is countable.

We must also check that $Q=\left(\tilde{x}_{1}, x_{2}, \ldots, x_{n}\right)$. Since $y_{1} \in Q$, we can write $y_{1}=\beta_{1} x_{1}+$ $\cdots+\beta_{n} x_{n}$ for some $\beta_{i} \in T$. Then we have

$$
\tilde{x}_{1}=x_{1}+\alpha_{1} y_{1}=\left(1+\alpha_{1} \beta_{1}\right) x_{1}+\alpha_{1} \beta_{2} x_{2}+\cdots+\alpha_{1} \beta_{n} x_{n} .
$$


Note that because $\alpha_{1} \in M$, the element $1+\alpha_{1} \beta_{1}$ is a unit. We can therefore write

$$
x_{1}=\left(1+\alpha_{1} \beta_{1}\right)^{-1}\left(\tilde{x}_{1}-\alpha_{2} \beta_{2} x_{2}-\cdots-\alpha_{1} \beta_{n} x_{n}\right)
$$

and conclude that $x_{1} \in\left(\tilde{x}_{1}, x_{2}, \ldots, x_{n}\right)$. Therefore we indeed have that $Q=\left(\tilde{x}_{1}, x_{2}, \ldots, x_{n}\right)$.

We now outline the process to obtain $R_{2}$. Let

$$
C_{2}=\left\{P \in \operatorname{Spec} T: P \in \operatorname{Ass}(T / r T), 0 \neq r \in R_{1}\right\} \cup \operatorname{Ass} T,
$$

which we note is a countable set. By Lemma 3.1, $M \notin C_{2}$ and $Q \not \subset P$ for all $P \in C_{2}$. We can therefore use Lemma 2.1 to find an element $y_{2} \in Q$ such that $y_{2} \notin P$ for all $P \in C_{2}$. Now fix an element $P \in C_{2}$ and let $D_{(P)}$ be a set containing one element of each coset of $T / P$ that makes $x_{2}+t y_{2}+P$ algebraic over $R_{1} /\left(P \cap R_{1}\right)$. Setting $D_{2}=\bigcup_{P \in C_{2}} D_{(P)}$, we can find $\alpha_{2} \in M$ such that $\tilde{x}_{2}=x_{2}+\alpha_{2} y_{2}+P$ is transcendental over $R_{1} /\left(P \cap R_{1}\right)$ for every $P \in C_{2}$. We can now let $R_{2}=R_{1}\left[\tilde{x}_{2}\right]_{\left(M \cap R_{1}\left[\tilde{x}_{2}\right]\right)}$. As above, $R_{2}$ is a countable N-subring and $Q=\left(\tilde{x}_{1}, \tilde{x}_{2}, x_{3}, \ldots, x_{n}\right)$.

We continue with this procedure to find $R_{3}, \ldots, R_{n}$, at each step setting $C_{i}=\{P \in$ $\left.\operatorname{Spec} T: P \in \operatorname{Ass}(T / r T), 0 \neq r \in R_{i-1}\right\} \cup \operatorname{Ass} T$, using Lemma 2.1 to find $y_{i} \in Q$ such that $y_{i} \notin P$ for all $P \in C_{i}$, and setting $D_{i}=\bigcup_{P \in C_{i}} D_{(P)}$, where $D_{(P)}$ is a set containing one element from each coset in $T / P$ that makes $x_{i}+t y_{i}+P$ algebraic over $R_{i-1} /\left(P \cap R_{i-1}\right)$. Through another use of Lemma 2.1, we find $\alpha_{i} \in M$ such that $\tilde{x}_{i}=x_{i}+\alpha_{i} y_{i}+P$ is transcendental over $R_{i-1} /\left(P \cap R_{i-1}\right)$ for every $P \in C_{i}$. We then set $R_{i}=R_{i-1}\left[\tilde{x}_{i}\right]_{M \cap R_{i-1}\left[\tilde{x}_{i}\right]}$.

We proceed thus for $i=3, \ldots, n$ to obtain the desired chain of $N$-subrings $R=R_{0} \subset$ $R_{1} \subset \cdots \subset R_{n}=S$, completing the proof.

We are now in a position to construct the desired UFD. As alluded to before, the proof of Theorem 3.4 is largely based on that of Theorem [2.6 from [1], which in turn is based on 
the proof of the main theorem in [5].

Theorem 3.4. For integers $n_{1}, n_{2}, \ldots, n_{k}$ with $2<n_{1}<n_{2}<\cdots<n_{k}$, there exists a local UFD A such that $A$ has maximal chains of prime ideals of lengths $n_{1}, n_{2}, \ldots, n_{k}$ which are disjoint except at their minimal and maximal elements.

Remark 3.5. As this proof is fairly long, we will preface it with a rough outline. We begin by using Lemma 3.1 to find a complete local ring $(T, M)$ in which we can find chains of prime ideals of the desired lengths. Our goal will be to use the construction in [5] to create a UFD $A \subset T$ such that $\widehat{A} \cong T$. We would also like $A$ to contain a generating set of each prime ideal in the aforementioned chains in $\operatorname{Spec} T$, as this would guarantee that their intersections with $A$ are all distinct. This can only be done for prime ideals $P$ such that ht $P>1$, however. We will deal with the case of the height one prime ideals separately.

To find such an $A$, it suffices to create an N-subring $R_{N}$ which contains a generating set of each prime ideal $P$ in the specified chains with ht $P>1$. This is accomplished through multiple applications of Lemma 3.3.

Finally, the height one prime ideals of the chains in $T$ must be dealt with. They are chosen such that their intersection with $R_{N}$ is $(0)$, which will help us ensure that they will all have the same intersection with $A$. Then other prime ideals can be found which make the chains disjoint, using Lemma 3.2. This will complete the proof.

Proof. First, using Lemma 3.1, let $(T, M)$ be a complete local ring satisfying the six conditions of Lemma 3.1. In particular, $T$ has minimal prime ideals $P_{1}, \ldots, P_{k}$ such that $\operatorname{dim}\left(T / P_{i}\right)=n_{i}$ for each $i$. Let $R_{0}$ be the prime subring of $T$ localized at its intersection with $M$ (note that $R_{0} \cong \mathbb{Q}$ ) and let $C_{0}=\operatorname{Ass} T=\operatorname{Min} T$. For each $P_{i} \in \operatorname{Min} T$, use Lemma 2.5 to construct a saturated chain of prime ideals $P_{i}=P_{i, 0} \subset P_{i, 1} \subset P_{i, 2} \subset \cdots \subset P_{i, n_{i}-1} \subset M$ such that each ideal in the chain contains exactly one minimal prime ideal and none (except $\left.P_{i, 0}\right)$ is an associated prime ideal of $T$. Note that the indices are chosen such that ht $P_{i, j}=j$. 
We will construct $A$ to be a local UFD such that $\widehat{A} \cong T$. We also want to ensure that, for $j>1,\left(P_{i, j} \cap A\right) T=P_{i, j}$. It is enough to have $A$ contain a generating set of each of these ideals $P_{i, j}$. We accomplish this through successive applications of Lemma 3.3. That is, we start with the N-subring $R_{0}$ and, say, the prime ideal $P_{1,2}$. By Lemma 3.3 , there exists a countable N-subring $R_{1} \supset R_{0}$ which contains a generating set for $P_{1,2}$. Applying this lemma in turn for each $P_{i, j}$ with $j>1$ results in a chain of countable N-subrings $R_{0} \subset R_{1} \subset \cdots \subset R_{N}$, where $R_{N}$ contains a generating set of each of the aforementioned prime ideals.

We now focus on the height one prime ideals of our chains. We will find prime ideals $\tilde{P}_{1}, \ldots, \tilde{P}_{k} \in \operatorname{Spec} T$ such that, for each $i=1, \ldots, k$, we have the following:

1. $P_{i, 0} \subset \tilde{P}_{i} \subset P_{i, 2}$,

2. $\tilde{P}_{i}$ contains exactly one minimal prime ideal of $T$, and

3. $\tilde{P}_{i} \cap R_{N}=(0)$.

Fix some $i \in\{1, \ldots, k\}$. We will show that, in fact, almost all prime ideals which satisfy (1) also satisfy (2) and (3). We begin by showing that there are uncountably many prime ideals that satisfy (1).

Suppose to the contrary that, for some fixed $i=1, \ldots, k$, the set $B_{i}=\left\{p \in \operatorname{Spec} T: P_{i, 0} \subset\right.$ $\left.p \subset P_{i, 2}\right\}$ is countable. Let $\tilde{T}_{i}=T_{P_{i, 2}} / P_{i, 0} T_{P_{i, 2}}$. The set $B_{i}$ is in one-to-one correspondence with the set $B_{i}^{\prime}=\left\{p \in \operatorname{Spec} \tilde{T}:(0) \subset p \subset P_{i, 2} \tilde{T}\right\}$, and so $B_{i}^{\prime}$ is countable. Hence, $\left|B_{i}^{\prime}\right|<$ $\left|\tilde{T} / P_{i, 2} \tilde{T}\right|$, so we can use Lemma 2.2 to conclude that $P_{i, 2} \tilde{T} \not \subset \bigcup_{p \in B_{i}^{\prime}} p$. But this is impossible, as every element in $P_{i, 2} \tilde{T}$ is contained in some height one prime ideal of $\tilde{T}$, which implies that in fact $P_{i, 2} \tilde{T} \subset \bigcup_{p \in B_{i}^{\prime}} p$. As a result of this contradiction, we conclude that $B_{i}$ is uncountable, as desired.

We now show that relatively few prime ideals satisfy (1) but not (2) and (3). Note that, given two distinct minimal prime ideals of a Noetherian ring, only finitely many height one 
prime ideals can contain them both. As a result, all but finitely many prime ideals satisfying condition (1) also satisfy condition (2). As for condition (3), note that, because $R_{N}$ is a countable subring of $T$, at most countably many height one prime ideals of $T$ will have nonzero intersection with $R_{N}$. To see this observe that, if we have $P \in \operatorname{Spec} T$ with ht $P=1$ and $0 \neq r \in P \cap R_{N}$, then $P \in \operatorname{Ass} T / r T$. As the set Ass $T / r T$ is finite for each $r$, the set $\left\{P \in \operatorname{Spec} T:\right.$ ht $\left.P=1, P \cap R_{N} \neq(0)\right\}$ is countable. There are hence uncountably many prime ideals $\tilde{P}_{i}$ that satisfy conditions (1), (2), and (3).

Let $\tilde{P}_{1}, \ldots, \tilde{P}_{k}$ be fixed prime ideals which satisfy conditions (1), (2), and (3) above. We now apply Lemma 2.1 with $I=\bigcap_{i=1}^{k} \tilde{P}_{i}$ and $C_{N+1}=\{P \in \operatorname{Spec} T: P \in \operatorname{Ass} T / r T, r \in$ $\left.R_{N}\right\} \cup$ Ass $T$ to conclude that there exists a nonzerodivisor $x \in I$ such that $x \notin \bigcup_{P \in C_{N+1}} P$. We now use a similar method to that in the proof of Lemma 3.3 to find an element $\tilde{x} \in T$ such that $R_{N+1}=R_{N}[\tilde{x}]_{\left(M \cap R_{N}[\tilde{x}]\right)}$ is a countable N-subring. Fix $P \in C_{N+1}$ and note that different choices of $t+P \in T / P$ give different values of $x(1+t)+P$, since $x \notin P$. Since the algebraic closure of $R_{N} /\left(P \cap R_{N}\right)$ in $T / P$ is countable, for all but countably many choices of $t \in T$, the image of $x(1+t)$ in $T / P$ will be transcendental over $R_{N} /\left(P \cap R_{N}\right)$. Now let $D_{(P)}$ be a set containing one element of each coset $t+P$ such that $x(1+t)+P$ is algebraic over $R_{N} /\left(P \cap R_{N}\right)$ and let $D=\bigcup_{P \in C_{N+1}} D_{(P)}$. Using Lemma 2.1, we can find an element $\alpha \in T$ such that $\tilde{x}+P=x(1+\alpha)+P$ is transcendental over $R_{N} /\left(P \cap R_{N}\right)$ for every $P \in C_{N+1}$. Finally, by Lemma 2.4, $R_{N+1}=R_{N}[\tilde{x}]_{(M \cap R[\tilde{x}])}$ is a countable N-subring. Note that, by the transcendental property guaranteed above, $\tilde{x} R_{N+1}$ is a prime ideal of $R_{N+1}$.

At this point, we can use the construction in [5], replacing $R_{0}$ in Theorem 8 of [5] with $R_{N+1}$ from above, to obtain a UFD $A \subset T$ such that $\widehat{A} \cong T$ and $R_{N+1} \subset A$. Therefore, $A$ contains a generating set for each $P_{i, j}$ with $j>1$ and, as a result, for each such ideal, $\left(P_{i, j} \cap A\right) T=P_{i, j}$. Therefore, letting $p_{i, j}=P_{i, j} \cap A$, for each $i=1, \ldots, k$ we have a chain of prime ideals $p_{i, 2} \subset p_{i, 3} \subset \cdots \subset p_{i, n_{i-1}} \subset M \cap A$ in $A$. Furthermore, the construction guarantees that prime elements in $R_{N+1}$ remain prime in $A$. Therefore, $\tilde{x} A \in \operatorname{Spec} A$ and 
$\tilde{x} A=\tilde{P}_{i} \cap A$ for each $i$.

We claim that $A$ in fact contains disjoint saturated chains of prime ideals as desired. To show this, we need only find appropriate height one elements $\tilde{p}_{i}$ such that, the chains (0) $\subset \tilde{p}_{i} \subset p_{i, 2} \subset \cdots \subset p_{i, n_{i-1}} \subset M \cap A$ are disjoint (except of course at (0) and $M \cap A$ ).

Now, using Lemma [3.2, we find height one prime ideals $\tilde{p}_{1}, \ldots, \tilde{p}_{k} \in \operatorname{Spec} A$ such that $\tilde{p}_{i} \subset p_{i, 2}$ for each $i=1, \ldots, k$, and $\tilde{p}_{i} \neq \tilde{p}_{j}$ whenever $i \neq j$. The chains $(0) \subset \tilde{p}_{i} \subset p_{i, 2} \subset$ $\cdots \subset p_{i, n_{i}-1} \subset M \cap A$ are therefore the desired maximal chains of prime ideals.

The main difference between the proof of Theorem 3.4 and that of Theorem 2.6 from [1] is that, in Theorem [2.6, it is enough to construct an N-subring containing a generating set of just one carefully-chosen prime ideal $Q$, which has the property that $\operatorname{dim}(T / Q)=1$ and ht $Q<\operatorname{dim} T-1$. This ensures that the resulting UFD $A$ will be noncatenary, but it gives us no further information about the structure of $\operatorname{Spec} A$. That we can apply this technique multiple times and obtain more information about $\operatorname{Spec} A$ is the primary insight of this result.

By virtue of Lemma 3.2, we have considerable control over the height one elements of the chains: for instance, we can leave them "collapsed," as they are before the application of the lemma. Alternatively, we can choose to apply the lemma for only some chains, leaving a few chains "collapsed" at height one and the rest disjoint (see Figure 3 for an example).

Corollary 3.6. Given distinct integers $n_{1}, n_{2}, \ldots, n_{k}$ such that $n_{i}>2$ for each $i=1, \ldots, k$, there exists a local UFD A with maximal chains of prime ideals of lengths $n_{1}, \ldots, n_{k}$ which share a height one element but all of whose height two elements and above are disjoint.

Furthermore, for any $\ell<k$, there are maximal chains of prime ideals of lengths $n_{1}, \ldots, n_{k}$ such that the first $\ell$ share a single height one element and the remaining $k-\ell$ are disjoint.

Proof. Construct $A$ as in the proof of Theorem 3.4. Then, the chains $(0) \subset \tilde{x} A \subset p_{i, 2} \subset$ $\ldots p_{i, n_{i}-1} \subset M \cap A$ are disjoint except at their maximal, minimal and height one elements. 
Finally, we note that by applying Lemma 3.2 to only $k-\ell$ chains, we can ensure that the first $\ell$ share a height one element and the rest are disjoint.

\section{Examples}

Figure 1: A poset $X$ with maximal chains of lengths $4,5,6$, and 7 , and the corresponding prime ideals in the rings $T$ and $A$ from Lemma 3.1 and Theorem 3.4 respectively.
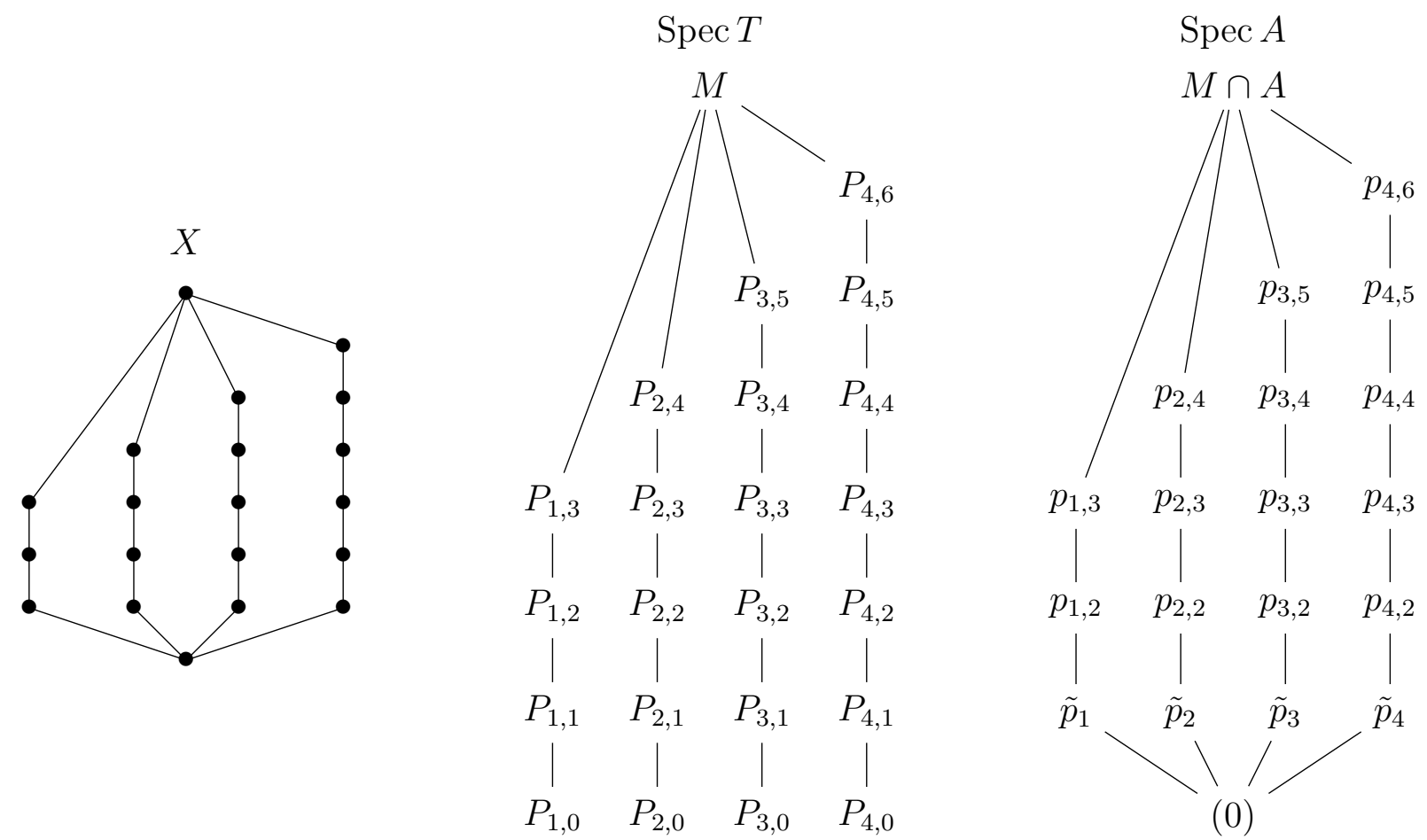

Example 4.1. Consider the poset $X$ as shown in Figure 1, which contains four maximal chains of lengths $4,5,6$, and 7 which are disjoint except at their minimal and maximal elements. As a result of Theorem 3.4, there exists a local UFD $A$ such that there is a dimension-preserving saturated embedding from $X$ into $\operatorname{Spec} A$. To find this ring $A$, we first apply Lemma 3.1 to find a complete local ring $T$ with chains of prime ideals as illustrated. 
We then apply Theorem 3.4 to find a local UFD $A$ which contains the illustrated prime ideals.

Figure 2: A partially ordered set which admits a saturated embedding, but no dimensionpreserving saturated embedding, into the prime spectrum of a Noetherian UFD $\left(X_{1}\right)$.
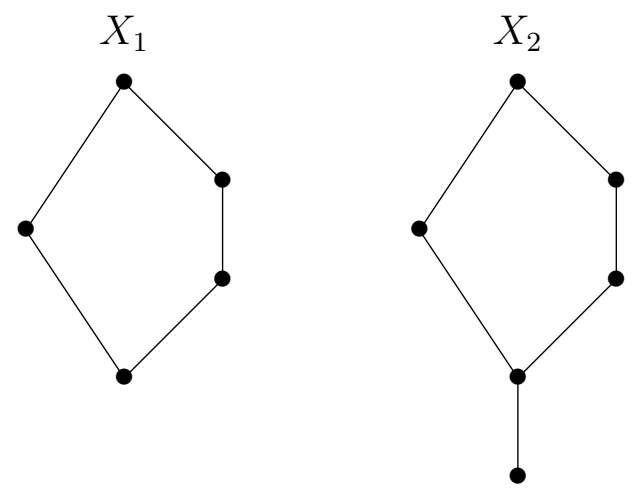

Example 4.2. Let $X_{1}, X_{2}$ be as in Figure 2, There is no dimension-preserving saturated embedding from $X_{1}$ to a local UFD, as every dimension-3 local UFD is catenary. However, as a result of Corollary 3.6 with $n_{1}=3$ and $n_{2}=4$, there is a dimension-preserving saturated embedding from $X_{2}$ to some local UFD. Therefore, for any local UFD $A$, every saturated embedding from $X_{1}$ into $\operatorname{Spec} A$ fails to preserve dimension.

Example 4.3. Let $Y_{1}, Y_{2}$, and $Y_{3}$ be the posets illustrated in Figure 3. As a result of Corollary 3.6, there exist local UFDs $A_{1}, A_{2}, A_{3}$ such that for $i=1,2,3$, there is a dimensionpreserving saturated embedding from $Y_{i}$ to $\operatorname{Spec} A_{i}$. 
Figure 3: Partially ordered sets with "disjoint" $\left(Y_{1}\right)$, "partially collapsed" $\left(Y_{2}\right)$, and "completely collapsed" $\left(Y_{3}\right)$ height one elements.
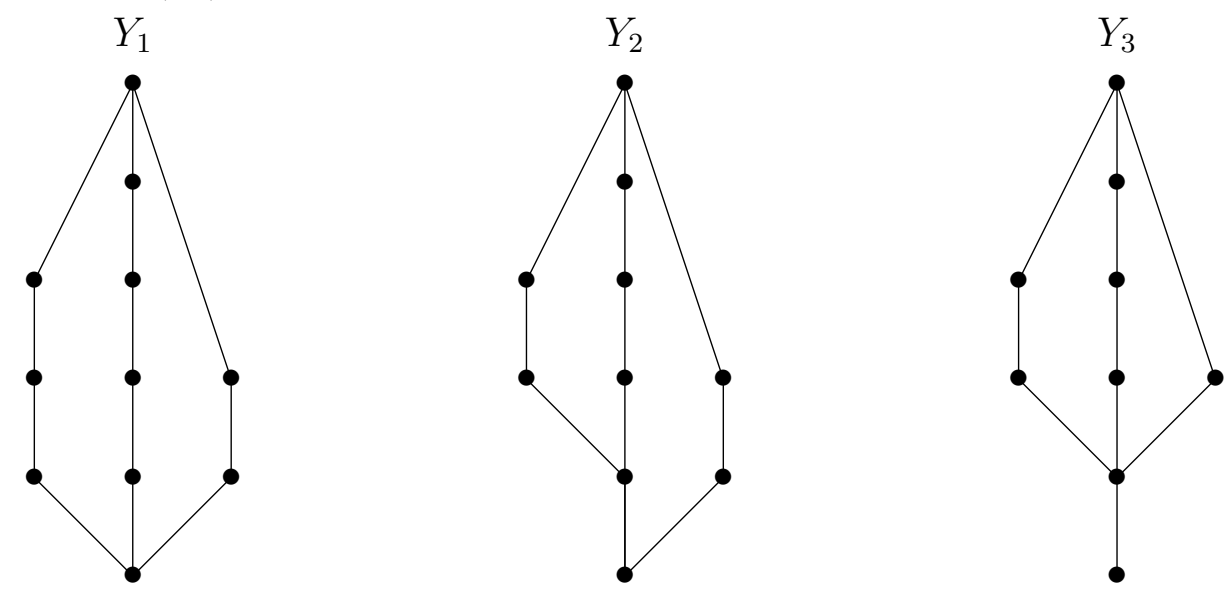

\section{Acknowledgements}

We would like to thank Andrew Bydlon for his many helpful comments, conversations, and suggestions. 


\section{References}

[1] C. I. Avery, C. Booms, T. M. Kostolansky, S. Loepp, and A. Semendinger. Characterization of Completions of Noncatenary Local Domains and Noncatenary Local UFDs. arXiv e-prints, September 2017.

[2] A. Dundon, D. Jensen, S. Loepp, J. Provine, and J. Rodu. Controlling formal fibers of principal prime ideals. Rocky Mountain J. Math., 37(6):1871-1891, 2007.

[3] R. Hartshorne. Algebraic Geometry. Graduate Texts in Mathematics. Springer New York, 2010.

[4] Raymond C Heitmann. Examples of noncatenary rings. Transactions of the American Mathematical Society, 247:125-136, 1979.

[5] Raymond C Heitmann. Characterization of completions of unique factorization domains. Transactions of the American Mathematical Society, 337(1):379-387, 1993.

[6] M. Hochster. Prime ideal structure in commutative rings. Transactions of the American Mathematical Society, 142:43-60, 1969.

[7] S. Loepp. Constructing local generic formal fibers. Journal of Algebra, 187(1):16-38, 1997.

[8] Hideyuki Matsumura. Commutative Ring Theory, volume 8. Cambridge university press, 1989.

[9] Masayoshi Nagata. On the chain problem of prime ideals. Nagoya Mathematical Journal, 10:51-64, 1956.

[10] R. Y. Sharp. Steps in Commutative Algebra. London Mathematical Society St. Cambridge University Press, 2000. 